\title{
Blinded Analysis of an Exercise ECG Database Using High Frequency QRS Analysis
}

\author{
Noam Omer ${ }^{1}$, Yair Granot ${ }^{1}$, Mika Kähönen ${ }^{2}$, Rami Lehtinen $^{3}$, Tuomo Nieminen ${ }^{4}$, Kjell Nikus ${ }^{5}$, \\ Terho Lehtimäki ${ }^{6}$ Jari Viik $^{7}$, Shimon Abboud ${ }^{8}$ \\ ${ }^{1}$ Biological Signal Processing Ltd, Tel-Aviv, Israel \\ ${ }^{2}$ Dept. Clinical Physiology, Hospital and Faculty of Med. \& Life Sci., Uni. of Tampere, Finland \\ 3Tampere Uni. of Applied Sci., Tampere, Finland \\ ${ }^{4}$ Dept. of Internal Med., Uni. of Helsinki, Helsinki, Finland \\ ${ }^{5}$ Heart Center, Tampere Uni. Hospital and Faculty of Med. and Life Sci., Uni. of Tampere, Finland \\ ${ }^{6}$ Dept. Clinical Chemistry, Fimlab Lab. and Faculty of Med. and Life Sci., Uni. of Tampere, Finland \\ ${ }^{7}$ BioMediTech Institute and Faculty of Biomedical Sci. and Eng., Tampere Uni. of Tech., Finland \\ ${ }^{8}$ Dept. of Biomedical Eng., Tel-Aviv Uni., Tel-Aviv, Israel
}

\begin{abstract}
High frequency QRS (HFQRS) analysis was shown to be more accurate than ST changes in detecting stress induced ischemia in designed clinical studies. Since it utilizes energy extracted from the frequency band of 150$250 \mathrm{~Hz}, \mathrm{HFQRS}$ analysis is performed with high end electrocardiographs which are equipped with adequate hardware. In this study, we aim to examine whether it is possible to perform the HFQRS analysis using ECG data acquired by a $500 \mathrm{~Hz}$ commercial electrocardiograph and also to assess the clinical performance of such analysis.

One hundred and thirty two ECG records of bicycle exercise tests were obtained from the FINCAVAS database. Fifteen records with a wide QRS duration and 28 patients who have not reached their target heart rate were excluded. HFQRS and computerized ST-segment analyses were performed for the remaining 89 records in a blinded fashion. Three records were excluded due to excessive high frequency noise. Accordingly, the group of records with a definite HFQRS interpretation included 57 patients without stenosis and 29 patients who had $>75 \%$ stenosis. Angiography, was used as gold standard. The clinical performance of both methods were assessed.

The HFQRS has statistically significant higher sensitivity of $86 \%$ comparing to the $41 \%$ of the ST $(p<0.005)$ with a statistically insignificant difference in specificity of $68 \%$ vs. $67 \%$ for the HFQRS and the ST respectively $(p=0.84)$.

This analysis demonstrated that HFQRS analysis may be applied for ECG data acquired by standard $500 \mathrm{~Hz}$ electrocardiographs and demonstrates its potential in diagnosing stress induced ischemia.
\end{abstract}

\section{Introduction}

For decades, ECG is the most commonly conducted cardiovascular diagnostic procedure and a fundamental tool of clinical practice [1]. The fact that ischemic heart disease (IHD) remains the leading cause of death globally [2] together with the availability of various beneficial treatment methods indicates that the ECG, as a safe,nonionizing and a low cost modality remains relevant for clinical practice, maybe even more than ever.

The analog frequency response and the sampling rate of electrocardiographs have a major effect on the sampled ECG signal. Although most of the conventional diagnostic information of the ECG is contained below $100 \mathrm{~Hz}$ in adults, it has long been recognized that higher-frequency components of the QRS complex (as high as $500 \mathrm{~Hz}$ ) may have clinical significance in patients with various forms of heart disease [3]. However, the current standard for diagnostic devices recommends a cutoff frequency of $150 \mathrm{~Hz}$ for the analog filter [1]. Many commercially available ECG devices apply a low pass filter at a cutoff frequency of $150 \mathrm{~Hz}$ or below. Such a filtration suppresses noise but it also attenuates the high frequency QRS (HFQRS) components.

ECG Stress Testing (EST) is widely used for an early diagnosis of IHD mostly in intermediate-risk patients. Since the fundamental principle of this test is to compare the patient's ECG signals at rest with its ECG signals at maximal physical exertion, the patient being examined must carry out a significant effort to complete the test. Accordingly, a test is usually diagnostic if $85 \%$ of the patient's maximal heart rate is achieved. However, EST is known to suffer from low accuracy with a sensitivity of 
45\%-50\% [4]. For this reason cardiac imaging modalities are often preferred.

High Frequency QRS analysis (HFQRS) was shown to be more accurate, in specifically designed clinical studies, in the detection of stress induced ischemia compared to conventional ST-segment analysis [5-8]. It was also mentioned in a scientific statement of the American Heart Association regarding EST standards as a useful tool for the detection of coronary artery disease (CAD) [10]. The physiological origin of the HFQRS was studied in a mathematical simulation [11] and in an animal model [1213]. Unlike conventional ECG analysis, which is based on changes in the repolarization phase of the cardiac cycle HFQRS analysis uses information from the depolarization phase (QRS complex). The independent nature of both analyses make HFQRS a beneficial tool in the diagnostic process since it adds new information that can be used in combination with the information from conventional analysis.

Since the quantitative diagnostic measure of an HFQRS analysis exists in the frequency band of $150-250 \mathrm{~Hz}$ [12], so far electrocardiographs with sampling rate of $1 \mathrm{kHz}$ and an adequate frequency response were used for this analysis. The fact that most standard electrocardiographs do not comply with such specifications has limited the use of HFQRS in clinical practice. In this study, we aimed to examine whether it is possible to perform an HFQRS analysis with ECG data recorded by a $500 \mathrm{~Hz}$ commercial electrocardiograph and to assess the clinical performance of such analysis.

\section{Methods}

\subsection{Analysis Group}

For this study 132 ECG records of bicycle exercise tests were obtained from the FINCAVAS database [14-17]. Tests were recorded with a CardioSoft system (GE Healthcare, Freiburg) at $500 \mathrm{~Hz}$. Fifteen records were excluded from the analysis according to the criteria for a maximal QRS duration of $120 \mathrm{~ms}$ ( 4 of them from the CAD group) as well as additional 28 patients who have not reached their target heart rate ( 24 from the CAD group). Accordingly, the analysis group comprised of 57 patients who had no stenosis (the reference group) and 32 patients who had $>75 \%$ stenosis in $\geq 1$ main vessel (the CAD group). Coronary angiography was used as gold standard.

\subsection{HFQRS Application}

The HFQRS analysis was performed with a commercial application (HyperQ, BSP, Tel-Aviv) which uses unique signal processing techniques for extracting the HFQRS signals from EST recordings. The HFQRS measurements are then used to diagnose the patient according to a specific decision rule which was previously shown to provide valuable diagnostic information for stress induced ischemia [6-8].

The extraction of these low amplitude HFQRS signals (measured in $\mu V$ ) is not trivial. First, a group of adjacent QRS complexes from a specific time segment are aligned. Then, the group is averaged to obtain its representative averaged QRS complex. A digital filter is then used for the filtration to the HFQRS frequency band. Thereafter, for quantification, the root mean square (RMS) of the HFQRS signal is calculated. This procedure is performed for every time segment throughout the test and its results are used to build the HFQRS time curve. This time curve describes the levels of the HFQRS over the course of the EST and is used for the derivation of the maximal relative and absolute reductions of each lead. Similarly to a conventional ECG analysis, the diagnosis of stress induced ischemia can be determined only for patients who have reached a sufficient level of exercise (i.e. have reached their target heart rate). An exception is the case where a patient has manifested significant HFQRS abnormalities even at lower heart rates. In this case the patient will be diagnosed as ischemic.

The criteria and thresholds for analyzing HFQRS signals for signs of ischemia were described in detail previously [6]. In short, a positive lead (i.e. a lead which indicates stress induced ischemia) is a lead where an absolute reduction of $\geq 1 \mu \mathrm{V}$ and a relative reduction of $\geq 50 \%$ were detected. For each test, three HyperQ interpretations are possible: ischemic, non-ischemic and non-diagnostic based on the following decision rule: A test with $\geq 3$ positive leads is considered ischemic. A test which complies with one of the following three possibilities is considered non-diagnostic: (1) A test with exactly 2 positive leads and $\geq 1$ noisy lead (i.e. a lead with excessive noise). (2) A test with exactly one positive lead and $\geq 2$ noisy leads. (3) A test without a positive lead and with $\geq 4$ noisy leads. Otherwise, a test is considered nonischemic.

\subsection{Diagnostic Performance Evaluation}

Each file from the analysis group was analyzed twice independently: once with the HFQRS application and once with the conventional ECG analysis. The HFQRS was performed in a blinded fashion whereas the criteria for the conventional ECG analysis were according to guidelines [4]. To assess the performance of the conventional ECG, a computerized ST-segment based on the values at rest and at peak exercise was used. Depression of $0.1 \mathrm{mV}$ or more from baseline was considered to be positive. For the evaluation of the clinical performance of both analyses, sensitivity and specificity were calculated using standard formulas. For the hypothesis testing a two-tailed Fisher's exact test was used and a p-value of $<0.05$ was considered statistically significant. 


\section{Results}

The HFQRS analysis produced HFQRS measurements which were similar to previous studies [12-13] in terms of: amplitude - order of magnitude was several $\mu \mathrm{V}$ and RMS levels - the vast majority of the values ranged from $1 \mu$ to $10 \mu$. An example for an averaged QRS complex and its corresponding high frequency waveform is presented in Figure 1. In this example it is noticeable that the energy of the HFQRS signal exist during the QRS complex where the signal fluctuates between $-10 \mu$ to $10 \mu$. The calculated RMS in this case is $3.5 \mu$.

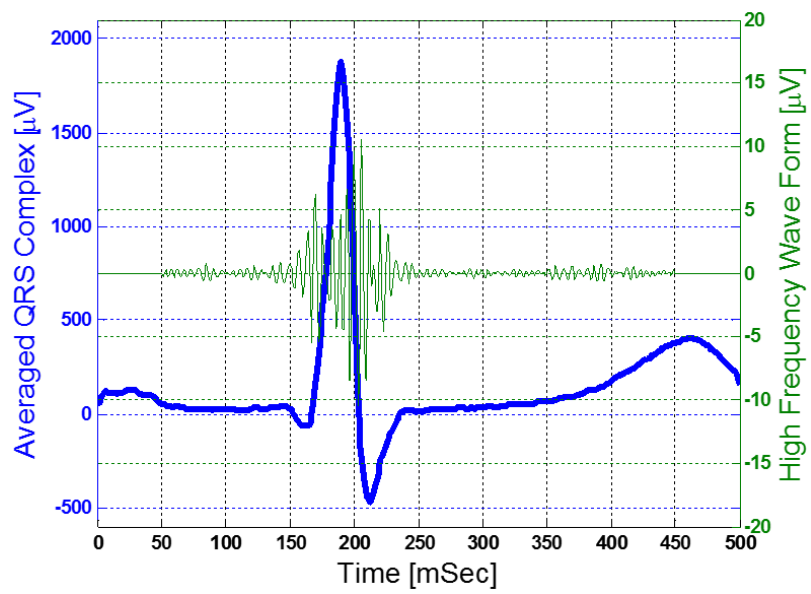

Figure 1. Example for an average QRS complex (marked with a thick line corresponds to the left y-axis) and its high frequency waveform derived from the filtration to the HFQRS band (marked with a thin line corresponds to the right $\mathrm{y}$-axis).

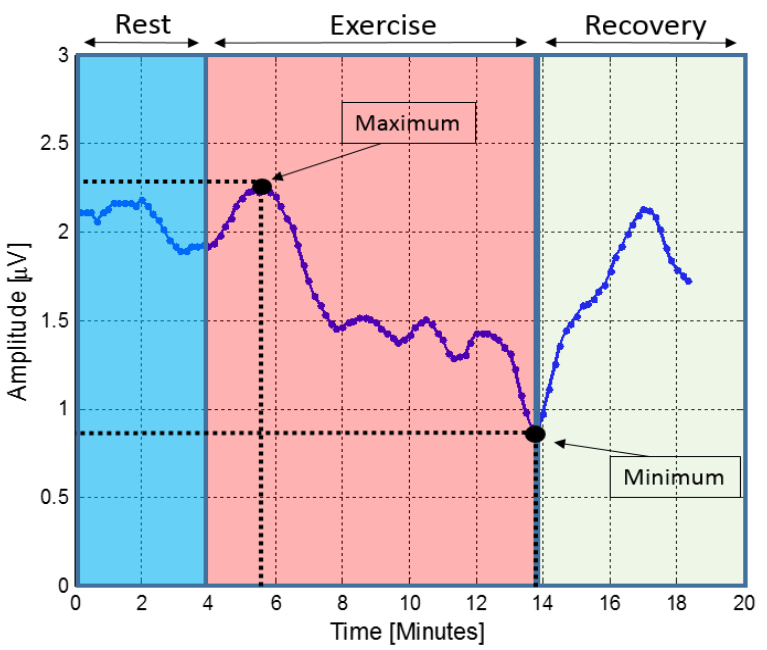

Figure 2. An example of a single HFQRS time curve derived from a $500 \mathrm{~Hz}$ commercial electrocardiograph recording and measured over the course of an exercise stress test. The different stages of the test are indicated as well as the maximal and minimal values.
In addition, a representative HFQRS time curve from one patient is illustrated in Figure 2. In this case, a relative decrease of $60 \%$ corresponding to an absolute reduction of $1.3 \mu \mathrm{V}$ was measured and thus the lead was declared positive.

Out of the 89 records in the analysis group, 3 records were excluded due to excessive high frequency noise. The remaining 86 tests included 57 patients who had no stenosis and 29 patients in the CAD group. The HFQRS showed statistically significant higher sensitivity of $86 \%$ comparing to $41 \%$ of the computerized ST analysis $(\mathrm{p}<0.005)$ with a statistically insignificant difference in specificity $(68 \%$ vs. $67 \%$ for the HFQRS and the ST respectively $(p=0.84))$. These results are demonstrated in Figure 3.

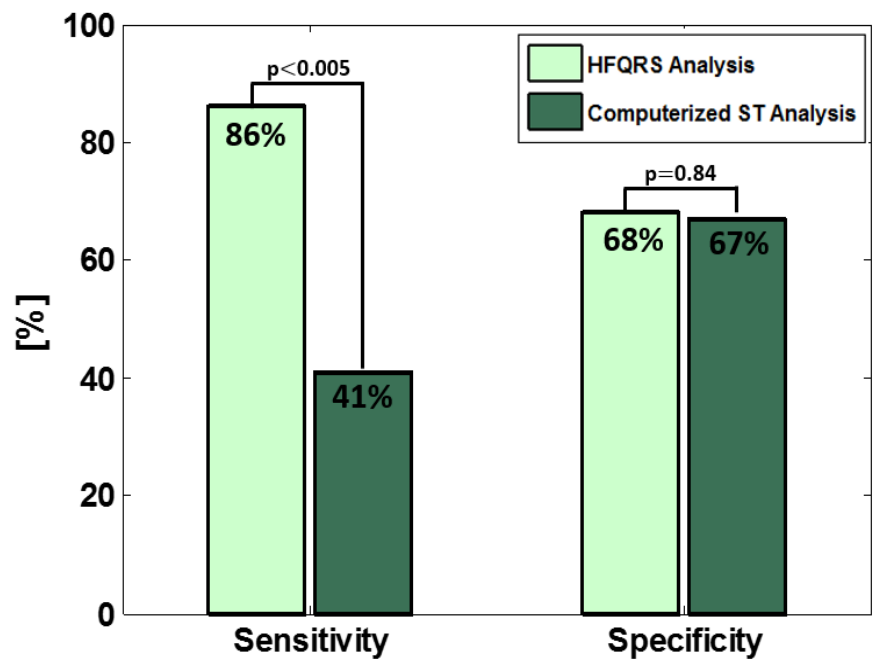

Figure 3. Diagnostic performance comparison between the HFQRS analysis and the computerized ST analysis in terms of sensitivity and specificity.

\section{Discussion}

In this study we examined whether it is possible to perform an HFQRS analysis for ECG data acquired using a commercial $500 \mathrm{~Hz}$ electrocardiograph. In addition, we wanted to test whether such analysis will produce valuable diagnostic performance even though the electrocardiograph does not meet the original requirements specified by the software.

To the best of our knowledge, this is the first time an HFQRS analysis is performed using a commercial $500 \mathrm{~Hz}$ electrocardiograph. The fact that the HFQRS signals were found to be measurable during a standard EST and were similar to signals presented in previous studies [12-13] is important by itself. In fact, it might be the first proof of concept which will change the conception that this analysis is relevant only for high end devices. Moreover, it may raise the motivation to embed such an analysis in devices 
which are more accessible and commonly used in clinics as an additional diagnostic tool enhancing the conventional ECG analysis.

The accuracy of HFQRS analysis presented in this study indicates the presence of valuable diagnostic information in the ECG data recorded at $500 \mathrm{~Hz}$, especially since the current analysis was performed in a blinded fashion. Having said that, we tend to believe that with appropriate adjustments these results might be even better. Moreover, the statistically significant difference in sensitivity, between the HFQRS and the conventional ECG, coincide with previous clinical evidence which indicate that HFQRS adds new information, originating from the depolarization phase, independent from the information which exists in the repolarization phase. Future studies may examine the optimal method for combining HFQRS analysis with conventional ECG analysis mainly since it may be performed retrospectively.

It is worth mentioning that the vast majority of the patients who have not reached a sufficient heart rate were ischemic and hence significantly shrunk the CAD group (decreasing it from 59 to 35). We do not consider this result as a deficiency of the HFQRS analysis because the inability of these patients to complete the test already implies to the presence of CAD. Nonetheless, a thorough investigation of these tests might yield a different analysis scheme which will enable the diagnosis of these tests.

In conclusion, when performing an HFQRS analysis, there is a significant importance that the HFQRS signals will not be filtered out during the acquisition process. Therefore, a device with an adequate analog frequency response and a sufficient sampling rate is a basic requirement. Furthermore, different specifications might lead to different HFQRS results and consequently different diagnostic performance. Thus, there are reasons to conduct a comprehensive study which will examine the compatibility of different electrocardiographs for an HFQRS analysis and will assess their clinical performance. This should be tested in a larger cohort, but the current study suggests that many commercial $500 \mathrm{~Hz}$ devices might be compatible for this analysis.

Since the FINCAVAS study contains records of thousands of patients and its results have already been published [14-17] we plan a future study to analyze the entire cohort with two major goals: (a) verifying the results of this study and past studies that were using the same software and (b) determining an optimal configuration for band limited ECG records which may enhance the performance of HFQRS for this specific configuration.

\section{References}

[1] Kligfield P., et al. Recommendations for the standardization and interpretation of the electrocardiogram: part I: the electrocardiogram and its technology. Circulation 2007;115(10):1306-24.
[2] WHO at: http://www.who.int/mediacentre/factsheets/ fs310/en. Updated on January 2017.

[3] Amit G., et al. Quantifying QRS changes during myocardial ischemia: Insights from high frequency electrocardiography. J Electrocardiol. 2014;47(4):505-11.

[4] Montalescot G., et al. ESC guidelines on the management of stable coronary artery disease. Eur Heart J. 2013;34(38):2949-3003.

[5] Pettersson J., et al. Changes in high-frequency QRS components are more sensitive than ST-segment deviation for detecting acute coronary artery occlusion. J Am Coll Cardiol 2000;36(6):1827-34.

[6] Sharir T., et al. Use of electrocardiographic depolarization abnormalities for detection of stress-induced ischemia as defined by myocardial perfusion imaging. Am. J. Cardiol 2012;109(5):642-50.

[7] Toledo E., et al. Detection of stress-induced myocardial ischemia from the depolarisation phase of the cardiac cyclea preliminary study. J Electrocardiol 2009;42(3):240-7.

[8] Rosenman D., et al. High-frequency QRS analysis improves the specificity of exercise ECG testing in women referred for angiography. J Electrocardiol 2013;46(1):1926.

[10] Gerald F., et al. Exercise Standards for Testing and Training A Scientific Statement From the American Heart Association. Circulation 2013; 128: 873-934.

[11] Abboud S., et al. Simulation of high-resolution QRS complex using a ventricular model with a fractal conduction system. Circulation Research 1991;68;1751: 60.

[12] Abboud S., et al. Detection of transient myocardial ischemia by computer analysis of standard and signal-averaged highfrequency electrocardiograms in patients undergoing percutaneous transluminal coronary angioplasty. Circulation 1987;76(3):585-96.

[13] Mor-Avi V., et al. Effects of coronary occlusion on highfrequency content of the epicardial electrogram and body surface electrocardiogram. Circulation 1987;76(1):237-43.

[14] Tuomo Nieminen N., et al. The Finnish Cardiovascular Study (FINCAVAS): characterizing patients with high risk of cardiovascular morbidity and mortality. BMC Cariovasc Disord 2006;6(9).

[15] Minkkenen M., et al. Prognostic capacity of c clinically indicated exercise test for cardiovascular mortality is enhanced by combined analysis of exercise capacity, heart rate recovery and T-wave alternans. 2015 Eur $\mathrm{J}$ Prev Cardiol;22(9):1162-1170.

[16] Pradhapan P., et al. Effect of heart rate correction on preand post-exercise heart rate variability to predict risk of mortality - an experimental study on the FINCAVAS cohort. 2014 Frontiers in Physiology;5(208):1-8.

[17] Hernesniemi J. A., et al. Predicting sudden cardiac death using common genetic risk variants for coronary artery disease. 2015 European Heart Journal;36(26):1669-75.

Noam Omer B.Sc, M.Sc

22a Wallenberg Street, Tel-Aviv, 6971918, Israel

Tel: +97236474840

Fax: +972 36471498

Email: noam@bsp.co.il 